\title{
Research Paper \\ Medical Procedure Problems From the Viewpoint of Elderly Referrals to Healthcare Centers of Hamedan: A Qualitative Study
}

Parisa Parsa ${ }^{1}$, Forouzan Rezapur-Shahkolai ${ }^{2}$, Maliheh Araghchian ${ }^{3},{ }^{*}$ Maryam Afshari ${ }^{4}$, Alireza Moradi ${ }^{4}$

1. Chronic Diseases (Home Care) Research Center, Hamadan University of Medical Sciences, Hamadan, Iran.

2. Research Center for Health Sciences, Hamadan University of Medical Sciences, Hamadan, Iran.

3. Department of Pharmacology and Toxicology, School of Pharmacy, Hamadan University of Medical Sciences, Hamadan, Iran.

4. Students Research Center, Hamadan University of Medical Sciences, Hamadan, Iran.

Received: 10 Jan. 2017

Accepted: 04 Apr. 2017

Key words:

Qualitative study,

Treatment mea-

sures, Drug, Elderly
Citation: Parsa P, Rezapur-Shahkolai F, Araghchian M, Afshari M, Moradi A. [Medical Procedure Problems from the Viewpoint of Elderly Referrals to Healthcare Centers of Hamedan: A Qualitative Study (Persian)]. Iranian Journal of Ageing. 2017; 12(2):146-155. http://dx.doi.org/10.21859/sija-1202146

dol: : http://dx.doi.org/10.21859/sija-1202146
Objectives Increasing age is associated with the risk of diseases and increased treatment problems. Due to the aging population and the need for consumption of drugs for chronic diseases of the elderly, understanding health challenges and treatment problems in the elderly is important. Based on this, a qualitative study was performed to identify treatment problems in older people's viewpoint in Hamadan . Methods \& Materials This qualitative study was conducted by content analysis approach. Data were collected through semi-structured propound interview with 15 elderly people having an age average of $66.47 \pm 4.47$ years, who were referred to health centers in Hamadan city in 2015 . Data was gathered by sampling based sexes with a maximum variation of age range of 60 to 75 years, socioeconomic status, and educational level. All interviews were simultaneously handwritten. Data were analyzed using content analysis method. Data strength was confirmed by participants and researchers.

Results Elderly patients had an average age of 66.47 years and a standard deviation of 4.47. Of the 15 participants, there were 6 females and 9 males. Data analysis resulted in extraction of 3 main themes "causes related to doctors, taking medication problems and shortage of drugs."These themes were determined by the following eleven subthemes: "the cost of visits to doctors, distrust of doctors, doctors not caring for the elderly, complications for the elderly, early completion medicines, efficacy, the absence of an alternative drug for existing drugs, many drugs for consumption, use of other drugs, lack of medicines in some pharmacies, and delivery of expired drugs to the elderly in some pharmacies".

Conclusion The results showed that there are problems of treatment in elderly. So after reviewing the problems with appropriate interventions, training can guide and help the elderly in this area. The authorities can also use findings from research in planning. 


\section{Extended Abstract}

\section{Objectives}

$\mathrm{n}$ recent years, the decline in fertility, along with increased longevity, has aged the world's population [1]. Moreover, improved living conditions, prevention of diseases, and provision of health services have affected the life expectancy and created a certain stage of life, defined as aging [2]. Aging is directly related to non-communicable diseases, increased medication use and therapeutic problems [3, 4]. Problems of medical procedure affect health, social and economic dimensions of the elderly [5]. Therefore, considering the importance and sensitivity of this issue as one of the challenges of national health system and the lack of appropriate scientific information about health issues of the elderly, this study was conducted to identify the problems of medical procedure from the viewpoint of the elderly referrals to healthcare centers of Hamedan during 2015.

\section{Methods \& Materials}

The present research was a qualitative study using qualitative content analysis [6] that was conducted on the elderly referrals to Hamedan healthcare centers in 2015. The research participants were selected through an objective-oriented sampling with maximum diversity. The study was approved by the Ethics Committee of Hamedan University of Medical Sciences with the code of ethics (UMSHA.REC.1394,2). Before the interview, the participants were informed about the project and the confidentiality of information. Interviews with the elderly were carried out in the education room of the healthcare centers with their consent and willingness.

Data were collected by the author through a semi-structured interview using the interview guide. All the interviews were conducted individually and face-to-face for approximately 30 to 40 minutes; all responses were literally taken down by another student. The inclusion criteria were elderly people aged between 60 and 75 years, taking medicine, and could participate in the study. Content analysis method was used to analyze the participants' responses. To do so, immediately after each interview, the word-by-word notes were examined more precisely by the research team and then recorded in the Word Software. The initial codes were allocated to the contents provided by the interviewees; in the next step, the similarities and differences were extracted from the concepts by constant comparison of the codes, so that a kind of merger was created on the same topics. In this way, the themes were extracted, and finally, the main topics of the study were obtained by reviewing the codes and themes.

\section{Results}

Of the 15 elderly participants, 6 were female and 9 were male. The use of diabetes medication was $26.5 \%$ higher in the participants than other medications. Three main concepts and 11 secondary concepts were extracted by

Table 1. Categorization of main and secondary contents from the medical procedure problems for the elderly

\begin{tabular}{|c|c|}
\hline Main Concepts & Secondary Contents \\
\hline \multirow{3}{*}{ Physician-related causes } & High cost of visiting physicians \\
\hline & Mistrust on physicians \\
\hline & Inattention of physicians to the elderly \\
\hline \multirow{7}{*}{ Medicine use problems } & Side effects of medicines for the elderly \\
\hline & Early running out of medicines \\
\hline & Effectiveness of medicines \\
\hline & \\
\hline & Lack of alternative medicines for existing ones \\
\hline & Multiple medicines to take \\
\hline & Using others' medicines \\
\hline \multirow{3}{*}{ Lack of medicines } & Lack of medicine in some pharmacies \\
\hline & \\
\hline & Delivery of expired medicines to the elderly at some pharmacies \\
\hline
\end{tabular}


data analysis. Medical procedure problems for the elderly include three main themes, namely physician-related causes (costs of visiting physicians, distrust of physicians, inattention of physicians to the elderly), medicine use problems (side effects of medicines for the elderly, early running out of medicines, effectiveness of medicines, lack of alternative medicines for existing ones, multiple medicines to take, using the others' medicines), and the lack of medications (lack of medicine in some pharmacies, delivery of expired medicines to the elderly at some pharmacies) (Table 1).

One of the factors affecting the use of medicines by the elderly is the high cost of doctors' visits. The elderly participants stated that they are reluctant to take medications given the inappropriate treatments and wrong prescriptions by some physicians. Most elderly people pointed out that doctors do not care for the elderly when prescribing medications and explaining drugs. The elderly claimed that their medications would run out soon, and it will affect the use of medicines.

Majority of the elderly stated that imported medications are much better than domestic one as it has a positive impact on their health. Some elderly people reported that their medications cause complications, suggesting that it would be better to find substitutes for medications. They also mentioned that taking one or two drugs is better than taking multiple drugs. Elderly people said they used others' medicines when their medication was over or they did not have access to medications. The elderly participants explained that the medicines are sometimes unavailable in the pharmacies. They also pointed out that some pharmacies provide them expired medications thinking them to be old with no proper knowledge.

\section{Conclusion}

According to the research results, the elderly participants suggested several problems for medical procedure, among which the physician-related causes, medicine use problems, and lack of medicines were the most important ones. Also, the elderly people pointed out that the healthcare centers of Hamadan had some problems related to medical procedure. The results mainly emphasize the challenges of medical procedure facing the elderly; proper training and interventions after examining the problems could help the elderly people in this field. Considering the importance of studies in this field and the lack of such studies, it can be one of the research priorities on aging, especially in scientific centers. Also, authorities can apply the research findings to appropriate planning and interventions for this group.

\section{Acknowledgments}

Hamedan University of Medical Sciences has financially supported the present paper.

\section{Conflict of Interest}

The authors declared no conflicts of interest. 


\title{
مشكلات اقدامات درمانى از ديدكاه مراجعان سالمندمراكز بهداشتىدرمانىشهر همدان (مطالعهاى كيفى)
}

\author{
بريسا هارسا'، فروزان رضايور شاهكلايى"، مليحه عراقجيان"، "مريم افشارى"، عليرضا مرادى"
}

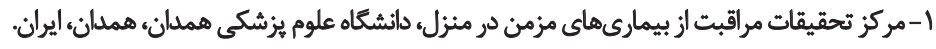

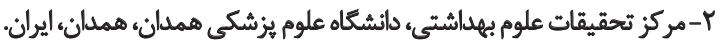

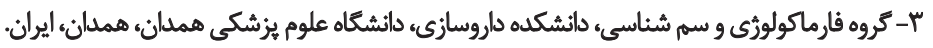

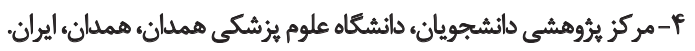

\begin{abstract}
حكدب

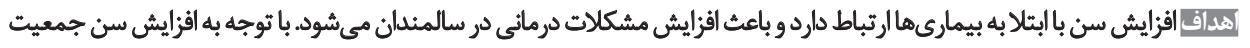

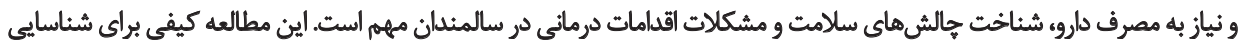

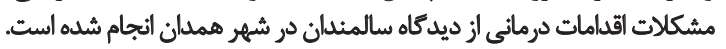

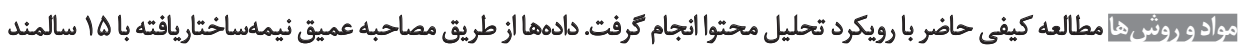

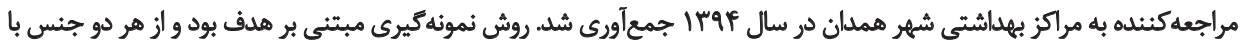

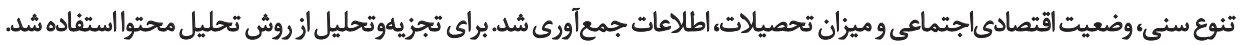

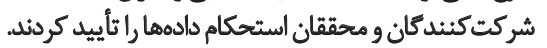

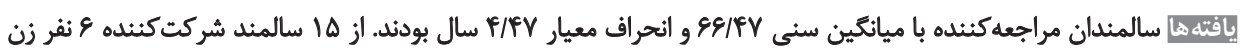

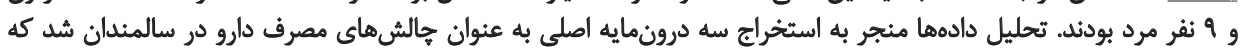

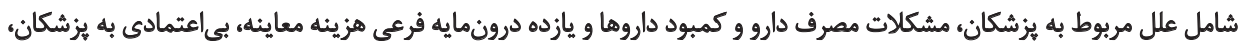

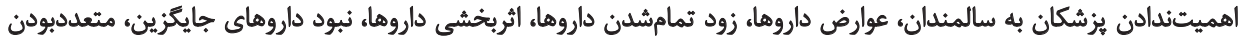

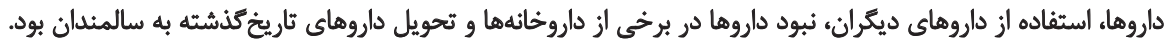

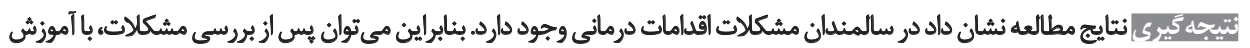

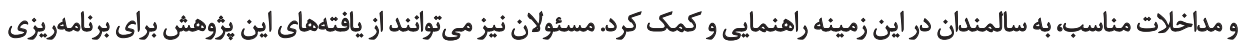

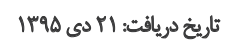

تاريخ بذيرش: 10

كشورهاى توسعهيافته تجربه شد، اما اخيراً در كشورهاى در حال

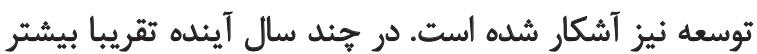

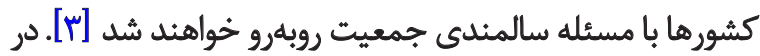

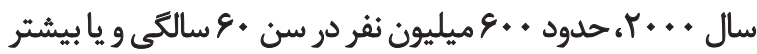

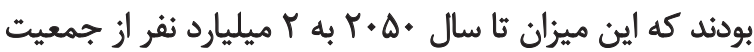

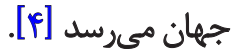

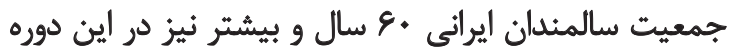
افزايش يافته است، بهطورى كه طبق دادههاي سازمان ،ئن آمار ايران،
مقدمه

در سال هاى اخير كاهش ميزان بارورى به همراه افزايش طول

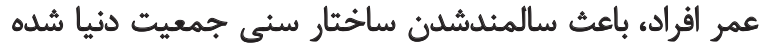

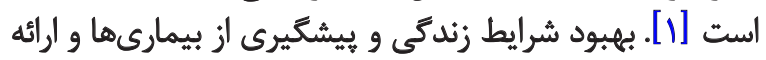

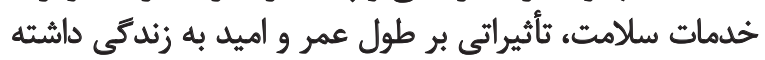

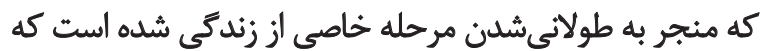

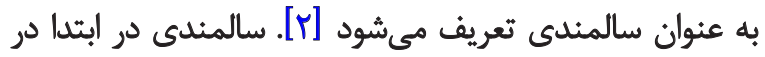




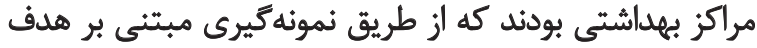

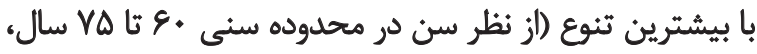

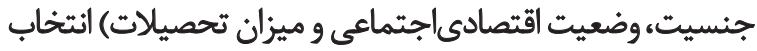

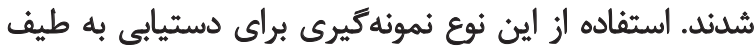

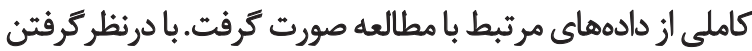

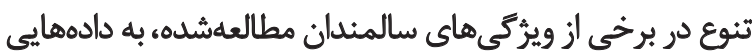
مناسب براى مطالعه مى توان دست بيدا كردي ساندان

اين مطالعه در كميته اخلاق دانشكاه علوم يُزشكى همدان

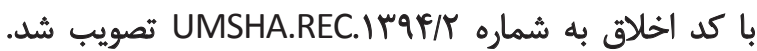

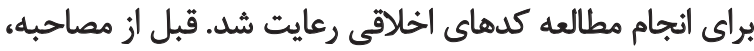

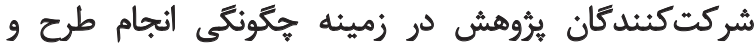

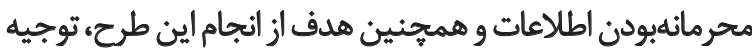

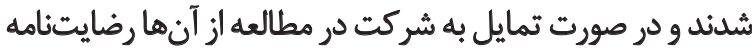
كتبى آكاهانه كرفته شد. مصاحبه با سالمندان با بار رضايت و و تمايل

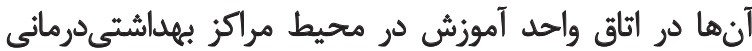

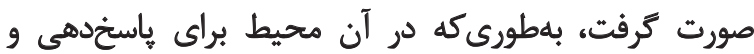

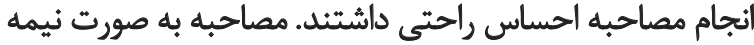

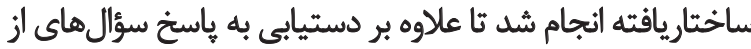

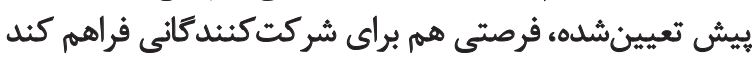

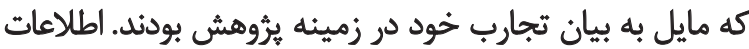

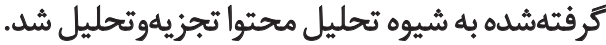

محقق دادها رالز طريق مصاحبه نيمه ساختاريافته با استفاده

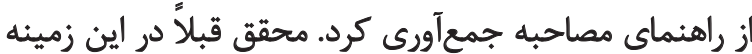

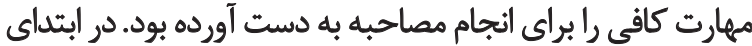

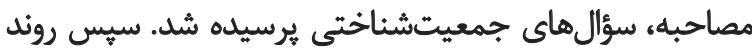

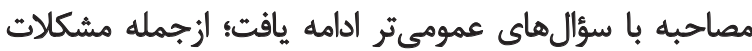

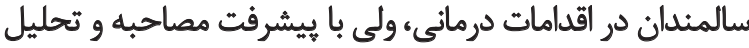

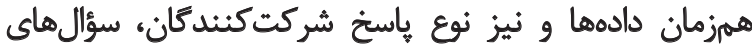

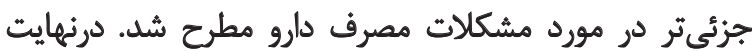

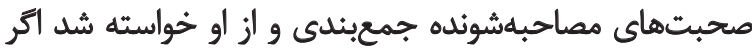
تناقضى وجود دارد آن را مطرح كند.

يك نفر از اعضاى تيم تحقيق كه دانشجوى دكتراى آموزش

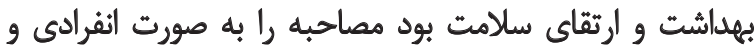

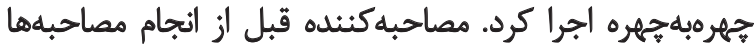

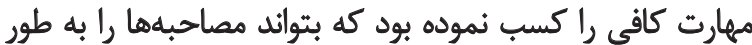

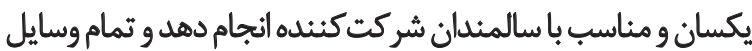

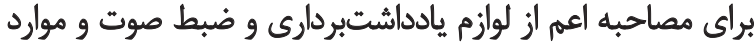

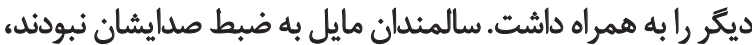

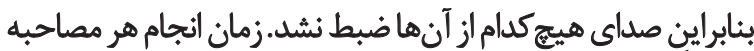

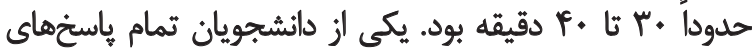

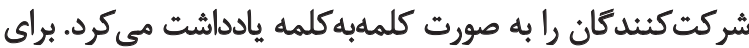

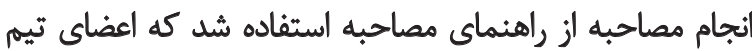

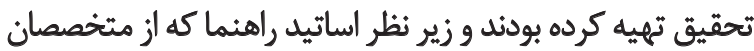

در سرشمارىهاى سالهاى

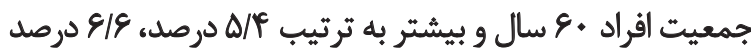

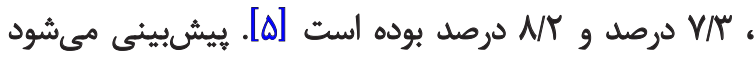

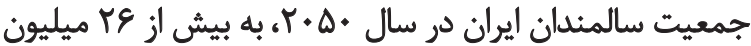

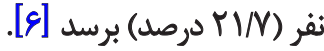
به دنبال افزايش جمعيت سالمندان، مشكلات سلامتي آنها نيز إنيز افزرايش و اهميت مىيابد. در بسيارى از سالمندان، كاهش ارئ ارتباطات

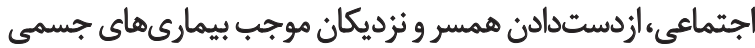

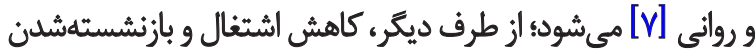

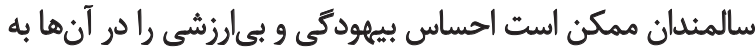

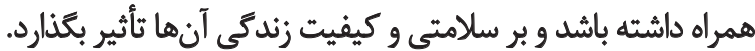

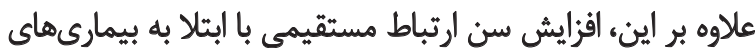

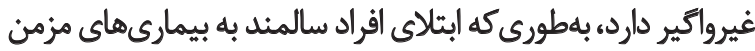

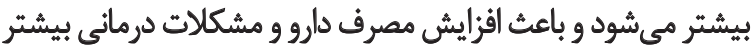

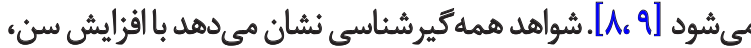

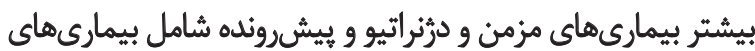

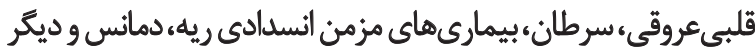

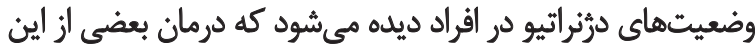

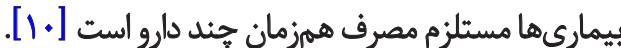
به دليل مشكلات زياد و بيمارىهاى ناشى از سالمندى، افراد

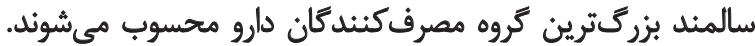

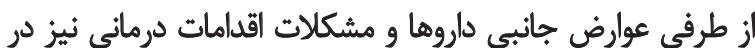

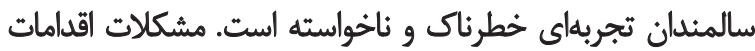

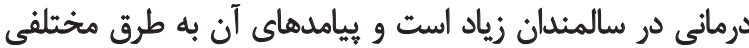

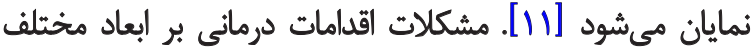

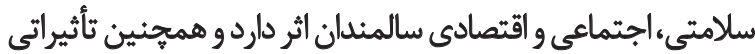

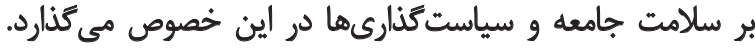

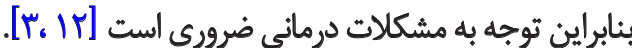

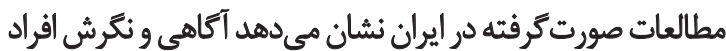

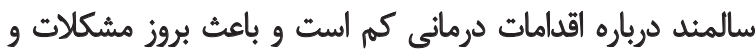

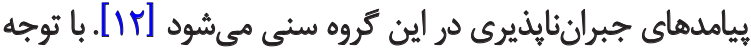

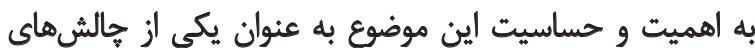

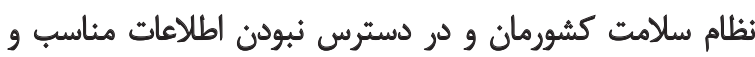

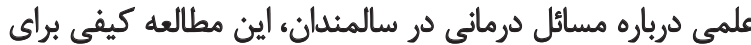

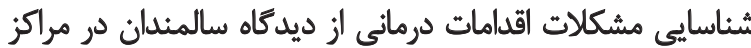

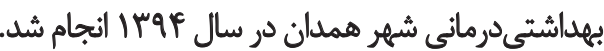

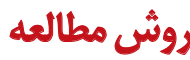

اين يروهش مطالعهاى كيفى أز نوع تحليل محتواى كيفى [بان]

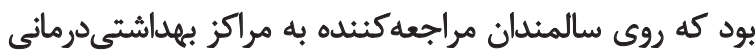

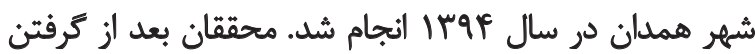

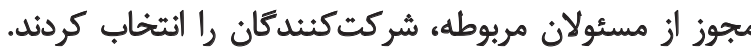

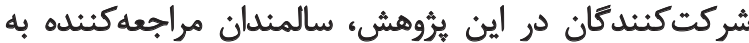


داروها براي سالمندان، زود تمامشدن داروها، اثربخشى داروها،

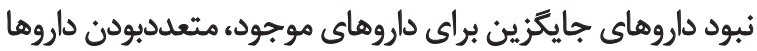

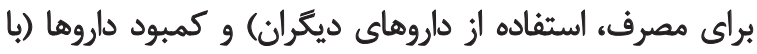

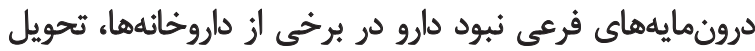

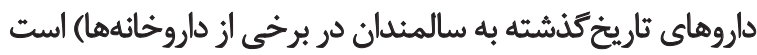

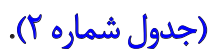

يكى از عواملى كه از نظر سالمندان روى مصرف داروهايشان اثر

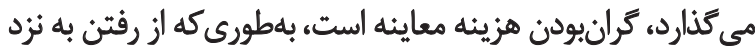

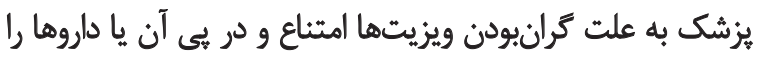

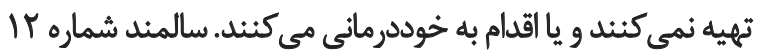

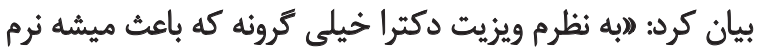
دكتر و خودم دارهامو بخرم كه بيشتر وقتام اشتباه مى كنمهان. سالمندان اظهار ميكردند كه به دليل درمانهاي نامناسب

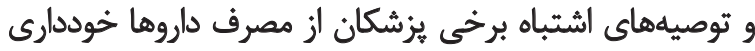

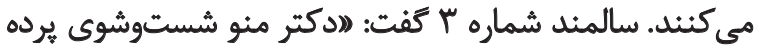

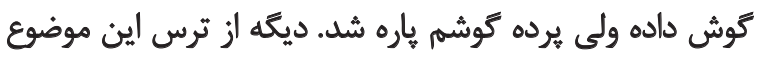

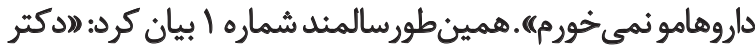

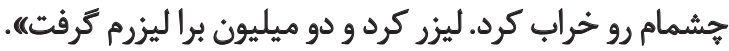

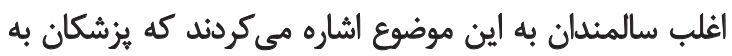

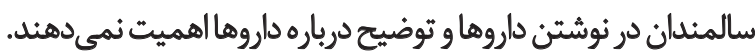

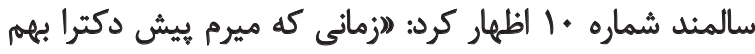

اهميت نميدن، حتى داروهامو جطورى بخورمهم بهم نميكنها.

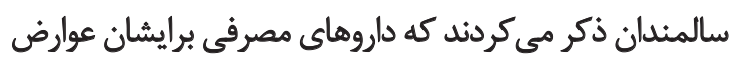

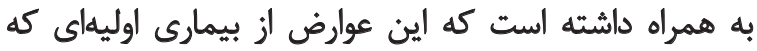

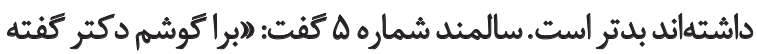

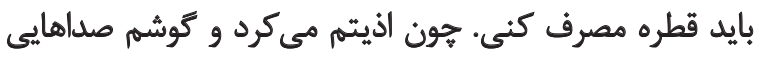
ازي مى اومد، منهم مصرفش رو قطع كردمهيه.

سالمندان شركت كننده در مطالعه ادعا داشتند كه داروهايشان

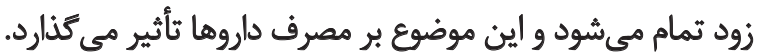

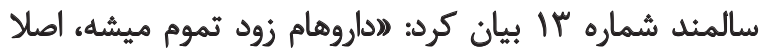

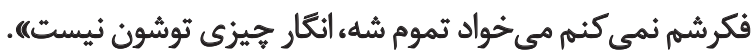

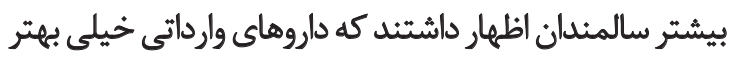

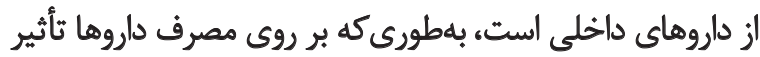

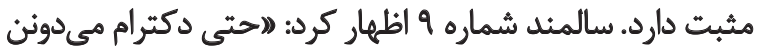

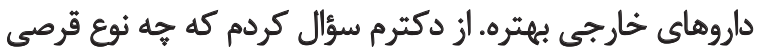

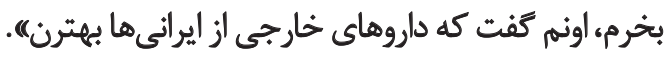
برخى از سالمندان بيان مىكردئد كه داروهايى كه مصرف

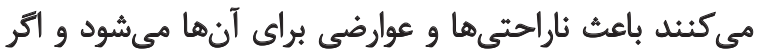

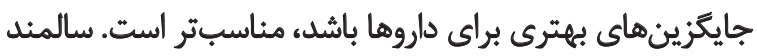

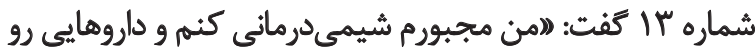

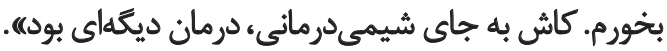

آموزش بهداشت و ارتقاى سلامت بودند بازنكرى شده بود.

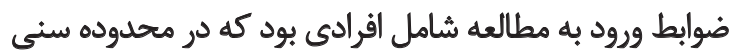

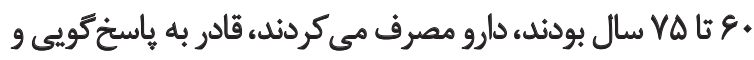

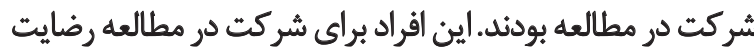

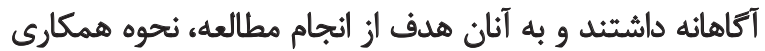

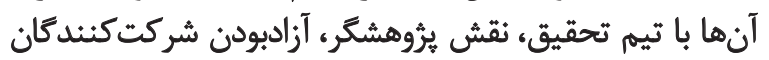

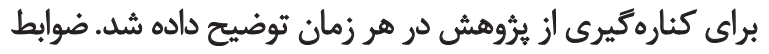

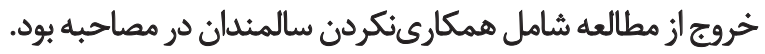
به منظور تجزيهوتحليل باسخهاى شركت كنيندكان از روش

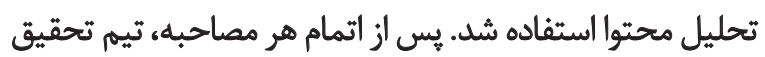

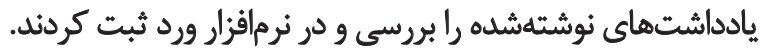

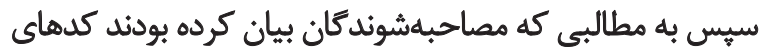

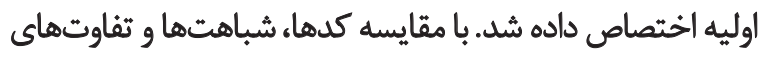

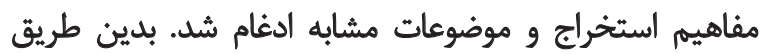

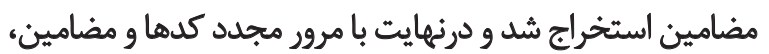

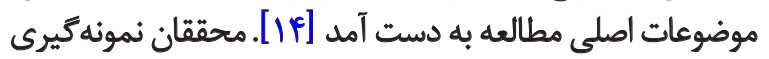

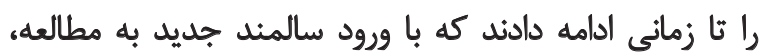

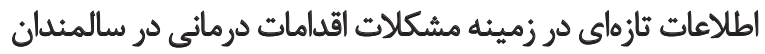

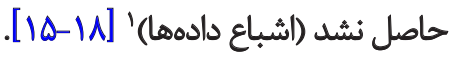
به منظور بالابردن دقت علمى و مؤثقبودن مطالعه براى ثأييد

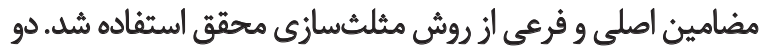

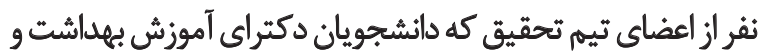

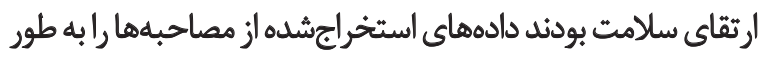

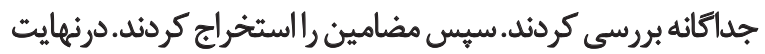

در مضامين اصلى و فرعى، تيم تحقيق به اجماع دست يافت.

ياثتهها

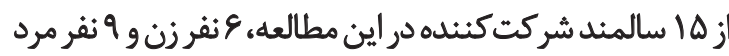

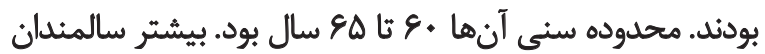

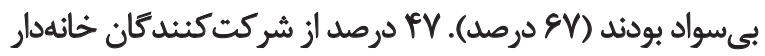

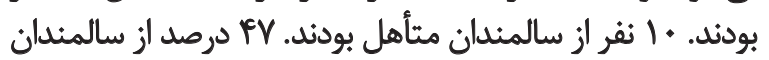

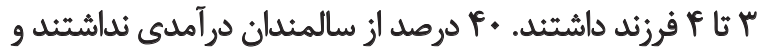

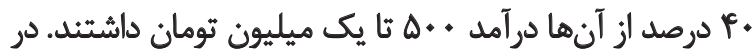

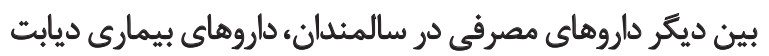

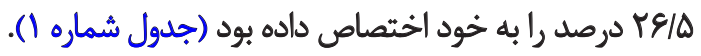
در اين برؤشش تحليل دادمها منجر به استخراج سه درونمايه

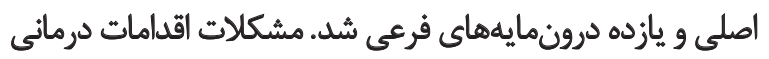

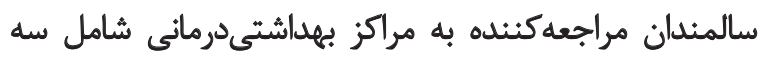

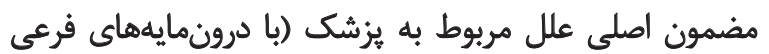

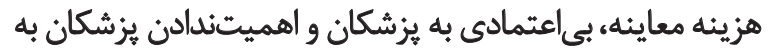

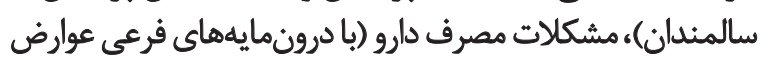


جدول ا. مشخصات جمعيتششناختى و زمينهاي شركت كنيندكان در مطالعه (ه نفر)

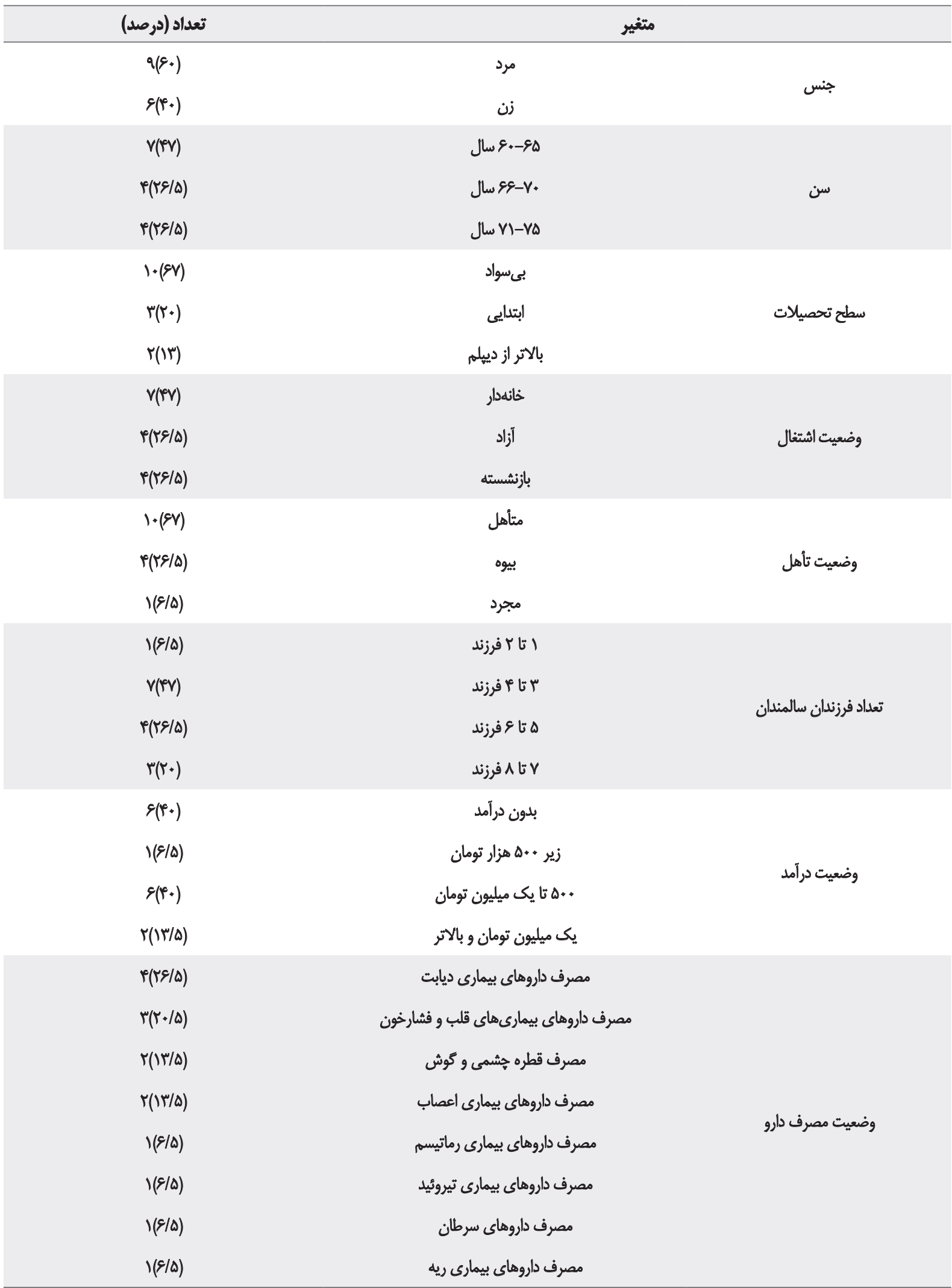


جدول Y. دستهنيندى درونمايهماي اصلى و فرعي از مشكلات اقدامات درماني سالمندان

\begin{tabular}{|c|c|}
\hline درون مايههاي فرعى & مفاهيم اصلى \\
\hline 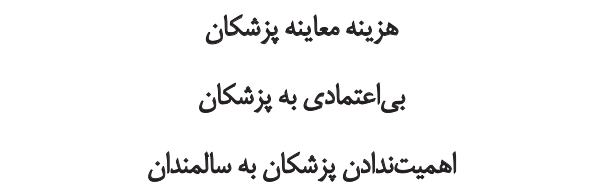 & علل مريوط به يزشك \\
\hline 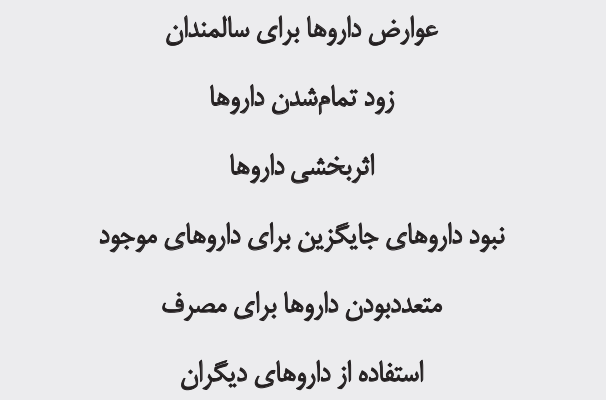 & مشكلات مصرف دارو \\
\hline 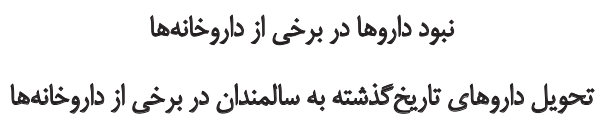 & كميود داروها \\
\hline
\end{tabular}

炾

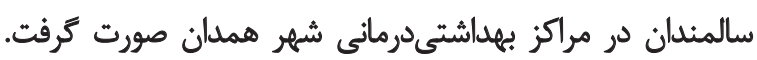

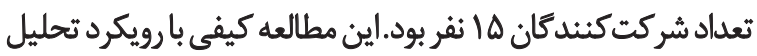

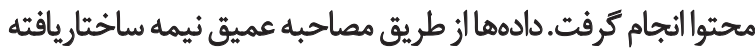

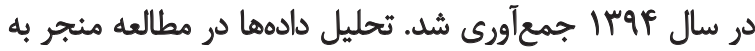

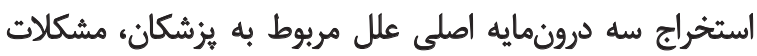
مصرف دارو و كمبود داروها و يازده درونمايه فرعى شد. مرند نتايج مطالعه نشان داد سالمندان براي مصرف دارو با مشكلاتى فري

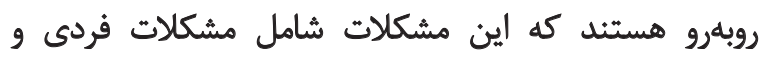

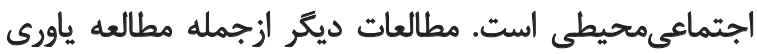

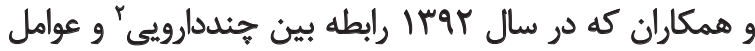
اجتماعى جمعيتشناختى رال رال در سالمندان آسايشعاه خيريه

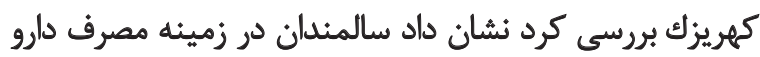

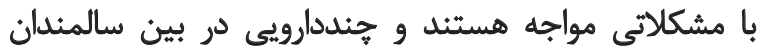

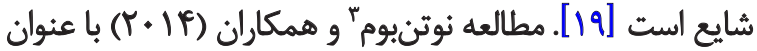

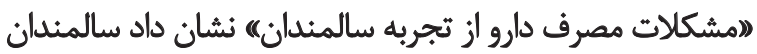

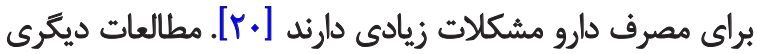

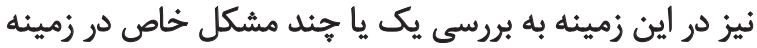

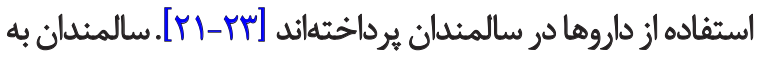

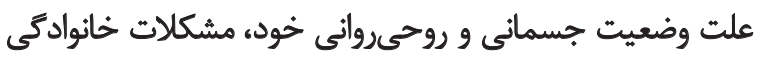

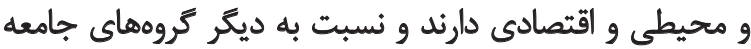

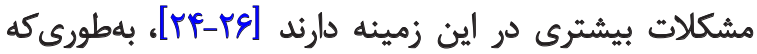

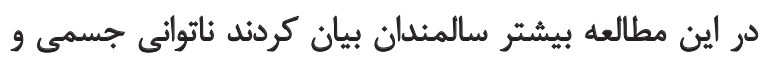

2. Polypharmacy

3. Notenboom
سالمندان ذكر هي كردند كه به علت متعددبودن داروها

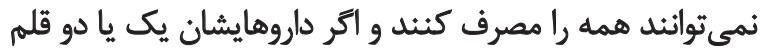

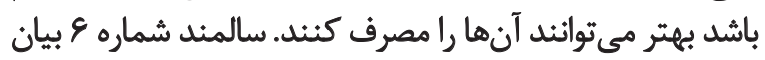

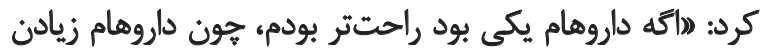

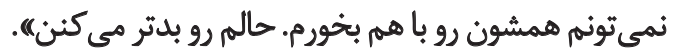
سالمندان شركت كنيده در مطالعه بيان مي كردند زمانى كه

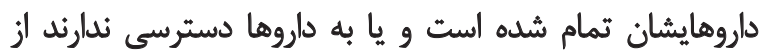

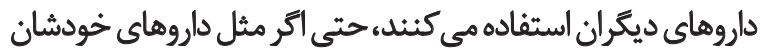

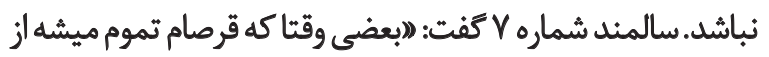

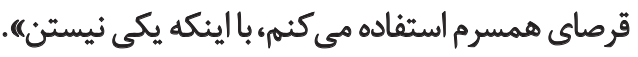
سالمندان شركت كنينده در مطالعه بيان مي كردند بعضى اوقات

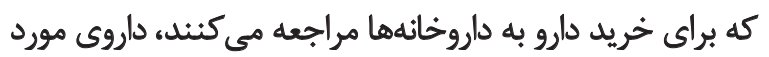

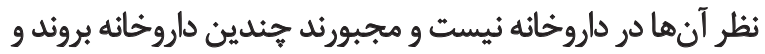

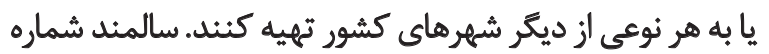

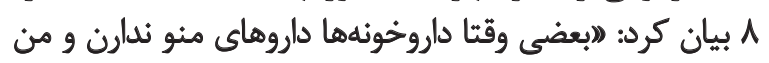

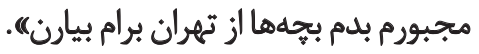
سالمندان به اين نكته اشاره مي كردند كه در برخى ازئ داروخائها متصدى داروخانه فكر مى كند آنها سالمند هستيند

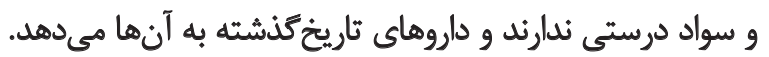

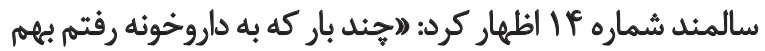

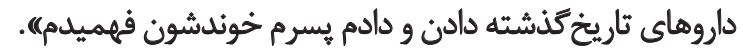
$\stackrel{\infty}{\leftrightarrow}$ مطالعه حاضر با هدف شئاخت مشكلات اقدامات درمانى از ديدگاه 
مشكلات مرتبط با اقدامات درمانى بودند. نتايج اين مطالعه به

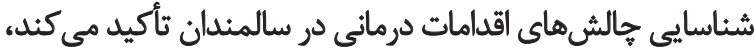

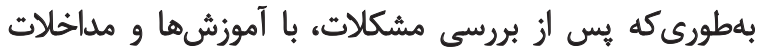

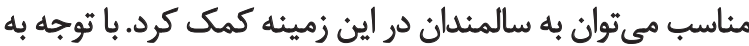

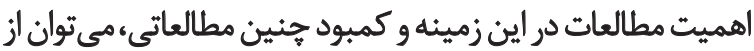

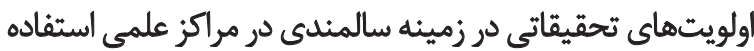

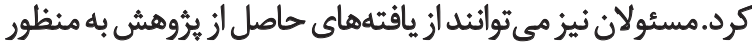
برنامهريزى و مداخلات مناسب براي اين تروه استفاده كنيند. از محدوديتهاى مطالعه مى توان به وجود ثفاوتهاي قومى،

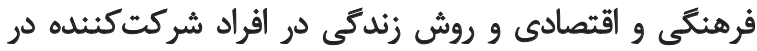

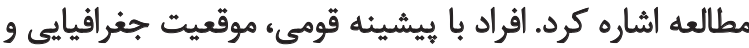

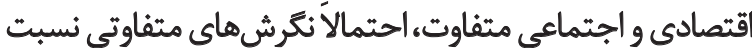

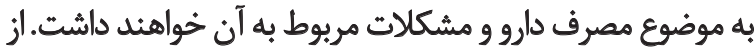

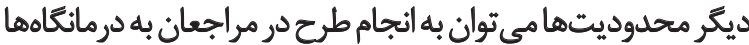

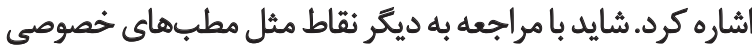
يا بيمارستانها نتايج كامل ترى به دست دسك مي آمدا مثل

$$
\text { تشكر وقدرداني }
$$

نويسندكان اين مقاله بر خود لازم مى دانند از دانشعاه علوم

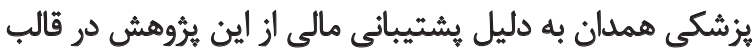

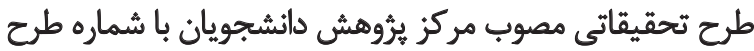
ئرج

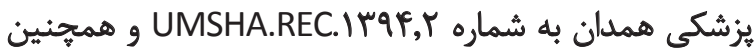

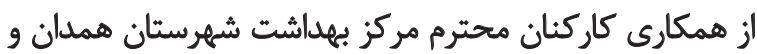

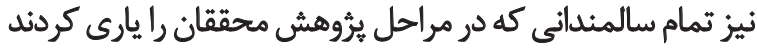
تشكر و قدرداني به عمل آورند.
ازكارافتادگى آنها تأثيرى منفى روى اقدامات درمانى آنها

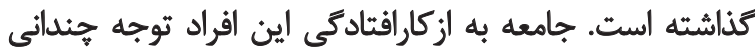

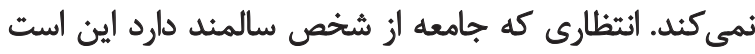

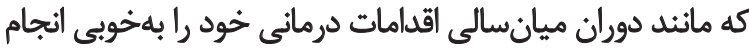

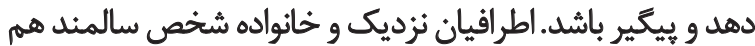

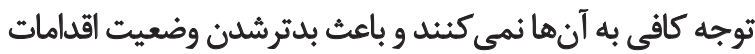

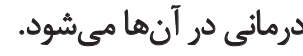

از ديكر مشكلات سالمندان اهميتندادن يُرشكان به سالمندان

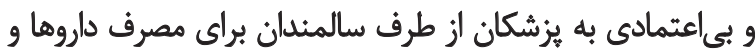

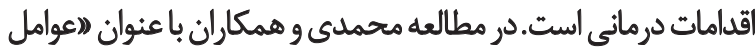
تسهيل

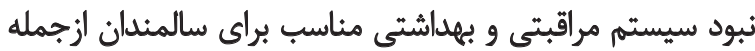

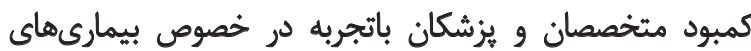
سالمندان، بر سلامت سالمندان تأثيركذار است [ـ ـ1].

بسيارى از سالمندان از بهتربودن داروهاى خارجى نسبت به به

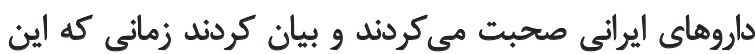

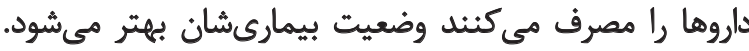

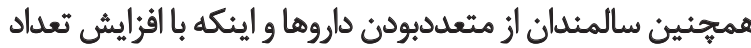

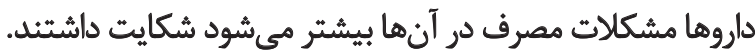

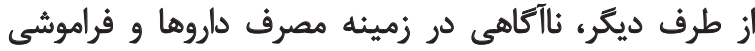

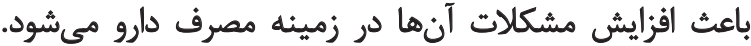

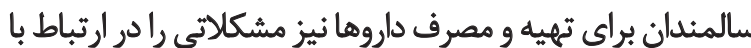

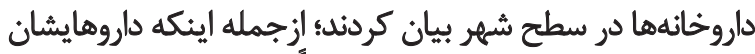

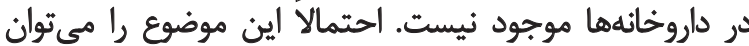

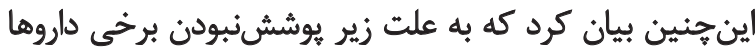

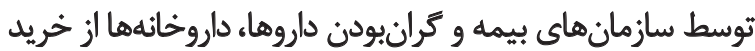

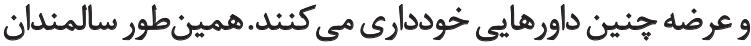

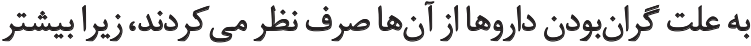

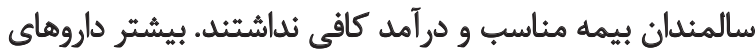

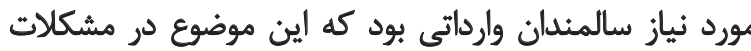

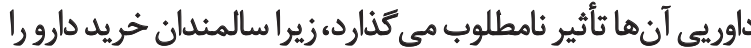

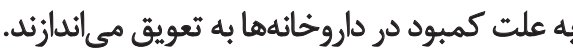
در مطالعه كيفى حاضر نقاط قوتى وجود داشت؛ ازجمله اينكه

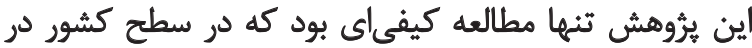

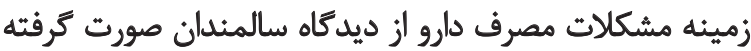

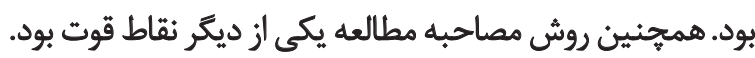

\section{نتيجهَيرى نهايى}

بر اساس نتايج حاصل از اين مطالعه، سالمندان شركت كنينده

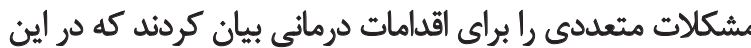

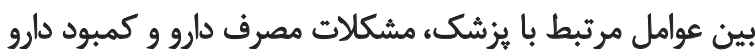

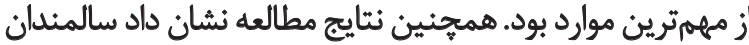

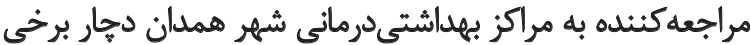




\section{References}

[1] Halter J, Ouslander J, Tinetti M, Studenski S, High K, Asthana S. Hazzard's geriatric medicine and gerontology. New York: Mc Graw Hill; 2009

[2] Abedi HA, Alavi M. [The experiences of health care team and elderly in-patients about concept of "elderly patients' education" in a hospital affiliated to Isfahan unifersity of medical sciences (Persian)]. Iranian Journal of Medical Education. 2007; 7(1):93-100.

[3] Salarvand S, Abedi HA. [Causes and motivations of elderly home residency from residents' point of view (Persian)]. Feyz Journals of Kashan University of Medical Sciences. 2008; 12(2):55-61.

[4] World Health Organization. Aging and life course [Internet] 2011 [Cited 2011 May 13]. Available from: http:/ / www.who.int/ ageing/age-friendly-world/en/

[5] Statistical Centre of Iran. [The overall results of the general census of population and housing 2006-2007 (Persian). Tehran: Statistical Centre of Iran; 2007.

[6] United Nations. World population ageing: 1950-2050 [Internet]. 2002 [Updated 2012 June 12]. Available from: http:/ / www. un.org/esa/population/publications/worldageing19502050/

[7] Bond J. Quality of life and older people. New York: McGraw-Hill Education; 2004

[8] Maas ML, Buckwalter KC, Hardy MD, Tripp-Reimer T. Nursing care of older adults: Diagnoses, interventions, and outcomes. Philadelphia: Mosby; 2001

[9] Rasel M, Ardalan A. [The future of ageing and its health care costs: A warning for health system (Persian)]. Iranian Journal of Aging. 2007; 2(2):300-5.

[10] Mohammadi Shah Bolaghi F, Dabaghi F, Yadavar Nikravesh M. [Facilitator and barrier factors in family caregiving process of iranian frail elderly: A qualitative study (Persian)]. Iran Journal of Nursing 2008; 21(55):55-65.

[11] Delshad Noghabi A, Darabi F, Baloochi Beydokhti T, Shareinia $\mathrm{H}$, Radmanesh R. [Irrational use of medicine status in elderly population of Gonabad (Persian)]. Quarterly of the Horizon of Medical Sciences. 2014; 19(5):49-56.

[12] SamadiS, Bayat A, Taheri M, Joneid BS, Roozbahani N. [Knowledge, attitude and practice of elderly towards lifestyle during aging (Persian)]. Journal of Qazvin University of Medical Sciences. 2007; 11(1): 83-84.

[13] Speziale HS, Streubert HJ, Carpenter DR. Qualitative research in nursing: Advancing the humanistic imperative. Philadelphia: Lippincott Williams \& Wilkins; 2011.

[14] Graneheim U, Lundman B. Qualitative content analysis in nursing research: Concepts, procedures and measures to achieve trustworthiness. Nurse Education Today. 2004; 24(2):105-12. doi: 10.1016/j.nedt.2003.10.001

[15] Molazade T, Honarbakhsh AR, Akrami AL, Tohidi I. [Report of exprience and function: Assessment of knowledge and practice in the field of consumption of food of elderly people of ages 60-69 in Esfarāyen 2009 (Persian)]. Paper presented at The $2^{\text {nd }}$ National Conference and The $1^{\text {st }}$ International Conference on Experiences and Good Functioning in Primary Health Care System. 30 October to1 November 2012; Bojnoord, Iran.
[16] Heidari Abedi H, Lali M, Keyvanniya S, Nikbakht A. [Life experiences of older people who are used to spend their time in parks (Persian)]. Journal of Qualitative Research in Health Sciences. 2013; 2(2):184-93.

[17] Morse J, Richards L. Read me first for a user's guide to qualitative research. New York: Sage Publications; 2002.

[18] Adib Hajbagheri M, Parvisi S, Salsali M. [Qualitative research methods (Persian)]. Tehran: Boshra; 2011.

[19] Yavari HR, Akbari Kamrani AA, Bakhshi E, Saboor M, Sahhaf R. [Association between polypharmacy and socio-demographic factors among elderly residents of Kahrizak charity foundation, Tehran, 2010-2011 (Persian)]. Iranian Journal of Ageing. 2014; 8(4):7-14.

[20] Notenboom K, Beers E, van Riet-Nales DA, Egberts TCG, Leufkens HGM, Jansen PAF, et al. Practical problems with medication use that older people experience: A qualitative study. Journal of the American Geriatrics Society. 2014; 62(12):2339-44. doi: 10.1111/jgs.13126

[21] Ruscin JM, Semla TP. Assessment of medication management skills in older outpatients. Annals of Pharmacotherapy. 1996; 30(10):1083-8. doi: 10.1177/106002809603001003

[22] Nikolaus T, Kruse W, Bach M, Specht-Leible N, Oster P, Schlierf G. Elderly patients' problems with medication. European journal of clinical pharmacology. 1996; 49(4):255-9. doi: 10.1007/ bf00226324

[23] Stegemann S, Ecker F, Maio M, Kraahs P, Wohlfart R, Breitkreutz J, et al. Geriatric drug therapy: Neglecting the inevitable majority. Ageing Research Reviews. 2010; 9(4):384-98. doi: 10.1016/j.arr.2010.04.005

[24] Philbert D, Notenboom K, Bouvy ML, van Geffen ECG. Problems experienced by older people when opening medicine packaging. International Journal of Pharmacy Practice. 2013; 22(3):2004. doi: 10.1111/ijpp.12070

[25] Tordoff J, Simonsen K, Thomson WM, Norris PT. It's just routine: A qualitative study of medicine-taking amongst older people in New Zealand. Pharmacy World \& Science. 2009; 32(2):154-61. doi: 10.1007/s11096-009-9361-5

[26] Van Geffen EC, Meuwese E, Philbert D, Bouvy ML. Problems with medicine packages: Experiences reported to a dutch medicine reporting system. Annals of Pharmacotherapy. 2010; 44(6):1104-9. doi: 10.1345/aph.1p052 